\title{
Numerical Investigation of the Impact of Shrinkage on the Pyrolysis of Biomass
}

\author{
Yuanyuan Lv ${ }^{1}$ Jihai Duan ${ }^{* 1,2}$
}

\author{
1College of Chemical Engineering, Qingdao University of Science and Technology, Qingdao, \\ 266042, P.R. China \\ ${ }^{2}$ State Key Laboratory Base of Eco-chemical Engineering \\ e-mail: Ivyuany2006@126.com, e-mail: duanjihai@yahoo.com
}

Keywords:shrinkage, MFIX, numerical simulation, biomass fast pyrolysis

\begin{abstract}
A new method for modeling shrinkage of a biomass particle is presented with the unreacted-core-shrinking model and shrinking core model for coal combustion. 2D model of biomass pyrolysis in a moving bed was established. The simulation was carried out with open source code MFIX by using Eulerian-Eulerian multiphase model. The results show that shrinkage can reduce the pyrolysis time, promote heat transfer, increase the biomass conversion percent and tar yield and decrease the non-condensable gas and char yield.
\end{abstract}

\section{INTRODUCTION}

Sustainable heat and power generation from biomass are paid the increasing attention owing to the increasing awareness about the continuous diminution in the availability of fossil fuels and the higher sensibility toward environment preservation from pollutants generated by conventional energetic systems [1]. A promising conversion method is the pyrolysis of cellulosic biomass [2-4]. For that, an elaborate understanding of how the chemical processes and transport mechanisms are altered by shrinkage, and particle size is needed [5,6]. In that way, establish appropriate and appropriate shrinkage model of degradation is significantly.

\section{BACKGROUND}

Many researchers have proposed some models for biomass pyrolysis [7]. The two primary aspects of these models are modeling the transport phenomena and modeling chemical reactions during pyrolysis. In these models, transport phenomena typically model gas flow within the solid of the particle in conjunction with energy conservation and gas and solid phase continuity. Most of these models assume one-dimensional mass and heat transport and local thermal equilibrium between the gas and solid species. Many of these models do not consider char shrinkage, resistance to flow within the particle, and recondensation of volatile species.

Parker has put forward a model that accounts for shrinkage by describing the change in geometry at the surface of a particle [8]. Parker predicts the heat release rates while flaming is occurring at the surface. The surface of the particle is assumed to contain no oxygen while flaming occurs. In this model, wood is composed of lignin, cellulose, and hemicellulose. First order kinetic parameters are determined for each of these components experimentally. Surface recession occurs in the char layer and the virgin wood according to this modified geometry. But this model accounts for shrinkage by changing the particle geometry at the exterior of a particle. Parker's model did not account for the difference of physical properties between char and wood.

A shrinking model of cellulose particle pyrolysis presented by Di Blasi [9] that accounts for heat, mass transport, and momentum. Internal gas flow is accounted for using Darcy flow with the permeability of the medium expressed as a function of char and wood content. This model uses a primary and secondary pyrolysis mechanism and variable solid densities. Shrinkage is defined as a function of three independent parameters. In this model the volume occupied by the solid decreases linearly with wood mass and increases with char mass multiplied by the solid shrinkage factor. This 
model illustrated that shrinkage resulted in a decrease in pyrolysis time and changes in the pyrolysis product yield. With a two-step reaction scheme and internal flow, this model was able to predict the products of pyrolysis. Properties continuous change is based on the respective amounts of wood and char using a linear progression variable. The model of Di Blasi provides increased flexibility. However, no experimental values are available for these parameters, and it is not clear whether the solid volume, wood porosity, and volatile volume have relationship of each other.

Based on unreacted-core-shrinking mode, a reaction-transport model is developed by Yenhan Lin et al. [12] for cellulose particles pyrolysis. Schematic diagram of a shrinking cellulose particle shows in Figure 1. The model contents experimental kinetic parameters, intra- particle and extraparticle heat transfer, and particle shrinkage. Mass of reactant and product are radius, time integral function by definition dimensionless parameter for unreacted cellulose, and shrinking factor of radius for active cellulose. Numerical predictions for measurable properties by this model can strongly agreement with experiments found. This model can predict mass of char, tar, noncondensable gas, unreacted cellulose under different time. But Lin's model is experimental model not consider the effect of momentum, mass transfer between air and solid, and only used for cellulose.

The work shown in this article is to simulate the effect of particle shrinking during the biomass pyrolysis proceeds, by coupling the unreacted-core-shrinking model and shrinking core model for coal combustion in MFIX. The schematic diagram of shrinking core model in MFIX is depicted in Figure 2[11]. The reaction rate expression is as follow.

$$
k=\frac{6 \varepsilon_{s m} P_{g}}{d_{p m}\left(\frac{1}{r_{f m}}+\frac{1}{r_{a m}}+\frac{1}{r_{m}}\right)}
$$

Where, $P_{g}$ is the partial pressure of gas, $\varepsilon_{s m}$ is the mth species volume fraction of solid phase. There are three kinds of resistance in the reaction rate: ${ }^{r}$ membrane resistance on the surface of the particles appearance; ${ }^{r m}$ surface reaction resistance and ${ }^{r_{m}}$ particle nuclear reaction resistance. In the process of biomass pyrolysis only considered surface reaction resistance, particle nuclear reaction resistance and through Sherwood number of particles (Sherwood number), and particle diameter on resistance parameters are calculated.
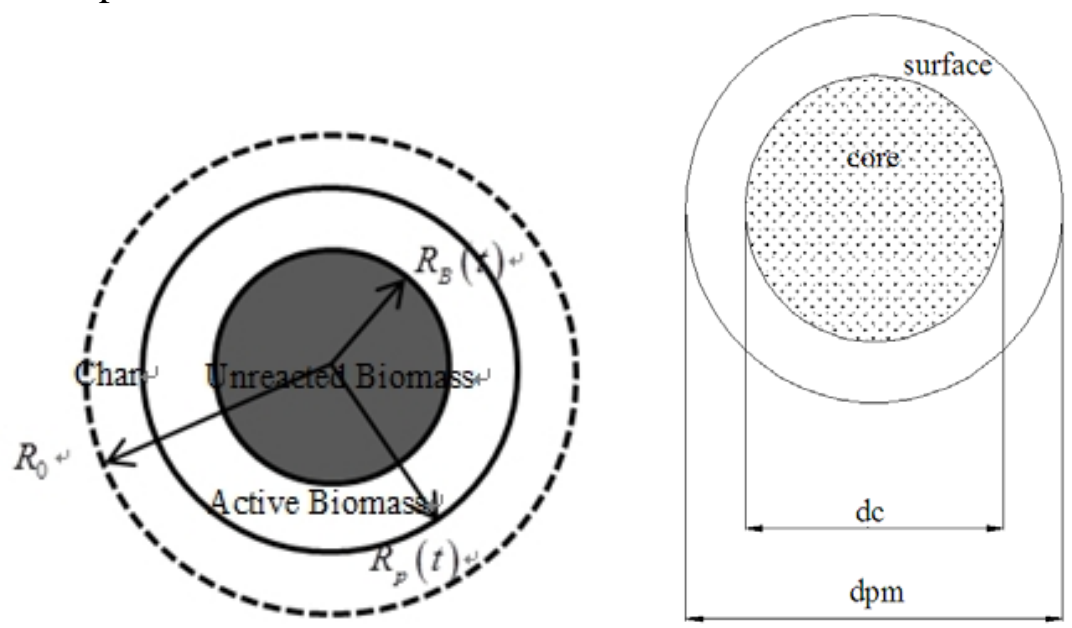

Figure 1. schematic diagram of shrinking cellulose particle. The initial radius is $\mathrm{R}_{0}$, the instantaneous radius is $R_{p}(t)$, and the effective, instantaneous radius of the unreacted cellulose is $R_{c}(t)$

Figure 2. Shrinking core model for coal combustion 


\section{MODEL DESCRIPTION}

In order to simplify the simulation, a furnace tube in the moving bed where biomass particle pyrolysis will carry out is used. The geometric model is illustrated in Figure 3 which has dimensions $5 \mathrm{~cm} \times 150 \mathrm{~cm}$. The reactor wall temperature is $1173 \mathrm{~K}$. The mesh size is $4 \mathrm{~mm} \times 4 \mathrm{~mm}$. The simulation and physical properties are given in Table I.

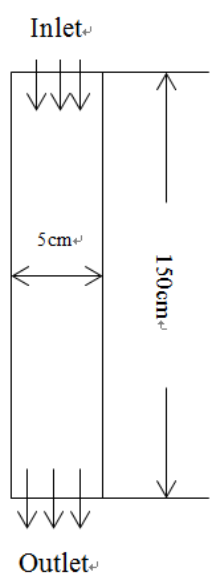

Figure 3. Schematic of a 2-D furnace tube for biomass pyrolysis

TABLE I. SIMULATION AND PHYSICAL PROPERITE

\begin{tabular}{|c|c|}
\hline \multicolumn{2}{|c|}{ Initial Condition } \\
\hline \multicolumn{2}{|c|}{ Value/comment } \\
\hline velocity of N2,biomass & $0.004 \mathrm{~g} / \mathrm{s} ; 1.7856 \mathrm{~g} / \mathrm{s}$ \\
\hline initial void fraction solid & 0.5 \\
\hline \multicolumn{2}{|c|}{ Particles } \\
\hline material & Bagasse \\
\hline mean diameter, d & $500 \mu \mathrm{m}$ \\
\hline density, $\rho \mathrm{s}$ & $400 \mathrm{~kg} \cdot \mathrm{m}-3$ \\
\hline
\end{tabular}

\section{RESULTS AND DISCUSSION}

Figure 4 shows the changing process of the instantaneous average tar mass fraction at the furnace tube outlet. At the beginning, because of the temperature of biomass particle is so low that the reaction rate of biomass is slow. So, there are little tar vapor at the furnace tube outlet. As the reaction time goes by, tar vapor gradually increases and the mass fraction is constant after the 20s. After stability, the average mass fraction of tar can reach $74.8 \%$ at the outlet. 


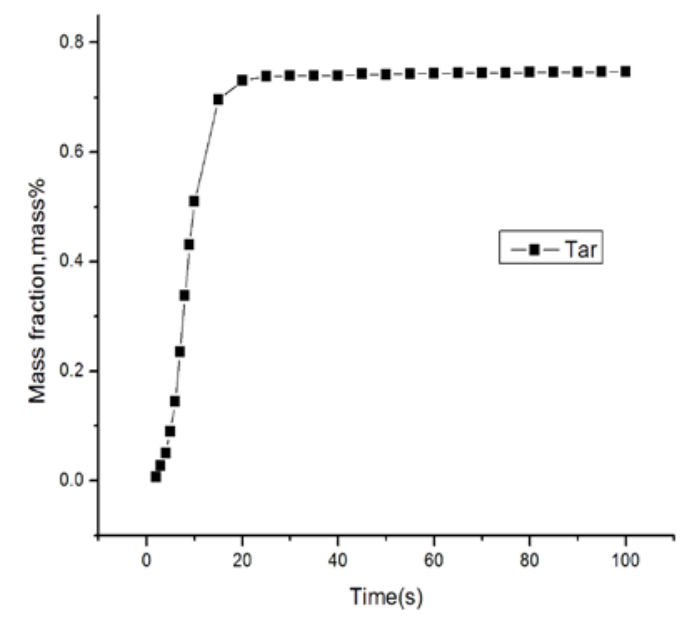

Figure 4. Variation of Tar mass fraction distribution in the furnace tube with time

Figure 5 shows the effect of particle shrinking on the yield of tar and conversion percent of biomass during bagasse pyrolysis. When the particle shrinkage is adopted in the simulation the higher conversion percent of biomass and tar yield will be obtained. During pyrolysis process, shrinkage was occurred at particle surface, as a result particle size decreased. This can effectively decrease the diffusion time of gas, reduce the secondary cracking of the tar vapor. That is, shrinkage is good for heat transfer. And it is of great importance to consider the effect of the particle shrinkage during the biomass pyrolysis reaction.

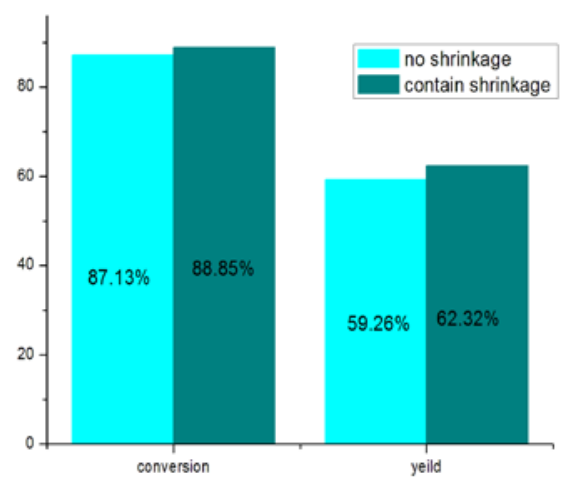

Figure 5. Comparison of conversion and yield of bagase and tar

Figure 6 illustrates the change trend of mass fraction of pyrolysis products along the reaction section axial height of furnace tube. It can be seen in this figure that at the beginning, the mass fraction of tar, gas, char are small. As the reaction progresses, the products gradually move towards the outlet. So, the mass fraction of products increases obviously and the mass fraction reaches maximum $74.6 \%$ of tar, $14.2 \%$ of gas, $10.5 \%$ of char respectively at the outlet. Compared with previous work, the tar yield is $4.35 \%$ higher than the old data showed in Figure 7 of no shrinkage, however, the gas and char yield is $2.50 \%$, and $1.52 \%$ less, respectively. 

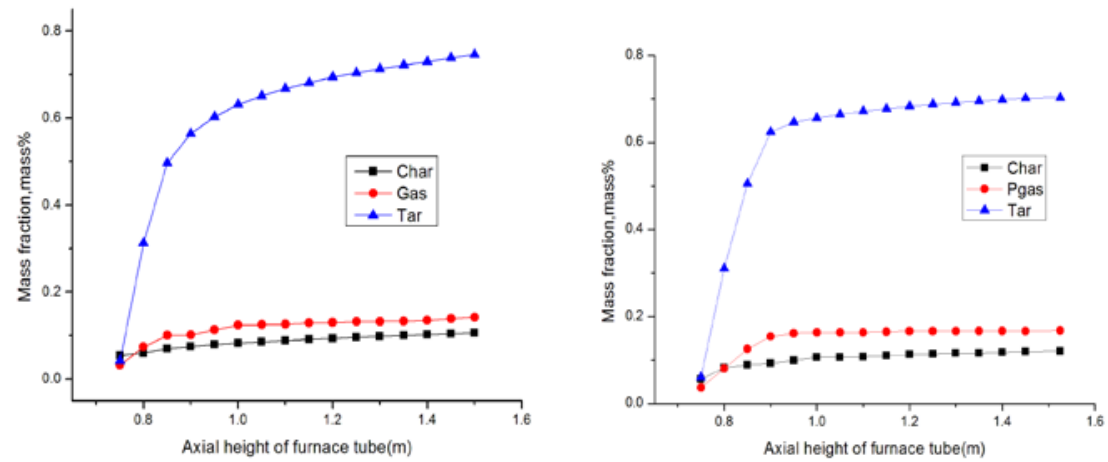

Figure6.Mass fraction distribution along reaction section axial height Figure 7.Mass fraction distribution along reaction section axial height

\section{CONCLUSION}

A new model for including particle shrinkage has been used in the numerical simulation of biomass pyrolysis. This shrinkage model shows the detail of the biomass pyrolysis to examine the impact of char shrinkage on the pyrolysis process. The results show that shrinkage has significant impact on pyrolysis time and product yield. The shrinkage of char can reduce the residence time of the pyrolysis gases in the moving bed reactor, and reduce the secondary thermal cracking of tar.

\section{Acknowledgment}

This work is supported by National Natural Science Foundation of China (No.21276132) and Livelihood Project Foundation of Qingdao (13-1-3-128-nsh).

\section{References}

[1] K.M. Bryden, and M.J. Hagge, Modeling the combined impact of moisture and char shrinkage on the pyrolysis of a biomass particle, Fuel, vol. 82, 2003, pp. 1633-1644.

[2] K.M. Bryden, and M.J. Hagge, Modeling the impact of shrinkage on the pyrolysis of dry biomass, Chem. Eng. Sci. vlo. 57, 2002, pp. $2811-2823$.

[3] Bridgwater, A Renewable fuels and chemicals by thermal processing of biomass, Chem. Eng. J. vlo.91, 2003, pp. 87-102.

[4] Elliott D., Historical developments in hydroprocessing bio-oils, Energy Fuels. Vlo. 21, 2007, pp. 1792-1815.

[5] K. Papadikis, H. Gerhauser, A.V. Bridgwater, and S. Gu. CFD modelling of the fast pyrolysis of an in-flight cellulosic particle subjected to convective heat transfer, Biomass and Bioenergy. Vol. 33, 2009, pp. 97- 107.

[6] K. Papadikis, S. Gu, and AV. Bridgwater, CFD modelling of the fast pyrolysis of biomass in fluidized bed reactors: Modelling the impact of biomass shrinkage, Chem. Eng. J. vol. 149, 2009, pp. 417-427.

[7] Lee J. Curtis, and Dennis J. Miller, Transport Model with Radiative Heat Transfer for Rapid Cellulose Pyrolysis, Ind. Eng. Chem. Vol. 27, 1988, 1775-1783.

[8] Parker, W. J., Prediction of heat release rate in wood. Ph.D. thesis, George Washington University, 1988

[9] Di Blasi, C., Heat, momentum, andmass transport through a shrinking biomass particle exposed to thermal radiation, Chem. Eng. Sci. vol. 51, 1996, 1121-1132. 
[10] Yenhan Lin, Joungmo Cho, Jeffrey M. Davis, and George W. Huber. Reaction transport model for the pyrolysis of shrinking cellulose particles. Chem. Eng. Sci. vol. 74, 2012, 160-171.

[11] M. Syamlal, W. Rogers, and T.J. O’Brien. MFIX documentation theory guide. US Dept of Energy, Technical Note DOE/METC-94/1004, 1993. 